\title{
Vector Flow Imaging Compared with Digital Subtraction Angiography for Stenosis Assessment in the Superficial Femoral Artery - A Study of Vector Concentration, Velocity Ratio and Stenosis Degree Percentage
}

\section{(두)(i) ()ㅇ $\Theta$}

\author{
Authors \\ Kristoffer Lindskov Hansen 1,2, Peter Møller Hansen', Caroline Ewertsen 1,2, Lars Lönn 1,3, Jørgen Arendt Jensen ${ }^{4}$, \\ Michael Bachmann Nielsen ${ }^{1,2}$
}

Affiliations

1 Department of Diagnostic Radiology, Copenhagen University Hospital, Copenhagen, Denmark

2 Department of Clinical Medicine, University of Copenhagen, Copenhagen, Denmark

3 Department of Vascular Surgery, Copenhagen University Hospital, Copenhagen, Denmark

4 Center for Fast Ultrasound Imaging, Technical University of Denmark, DTU Elektro, Kgs. Lyngby, Denmark

Key words

vector flow imaging, vector concentration, velocity ratio, digital subtraction angiography, peripheral arterial disease

$\begin{array}{ll}\text { received } & 19.09 .2018 \\ \text { revised } & 01.12 .2018 \\ \text { accepted } & 29.01 .2019\end{array}$

Bibliography

DOI https://doi.org/10.1055/a-0853-2002

Published online: 2019

Ultrasound Int Open 2019; 5: E53-E59

(c) Georg Thieme Verlag KG Stuttgart · New York

ISSN 2199-7152

Correspondence

Dr. Kristoffer Lindskov Hansen, MD

Department of Diagnostic Radiology

Copenhagen University Hospital

Blegdamsvej 9

DK-2100 Copenhagen

Denmark

Tel.: + 45/354/53 545

lindskov@gmail.com

\section{ABSTRACT}

Purpose Stenosis of the superficial femoral artery (SFA) induces complex blood flow with increased velocities. Disease assessment is performed with Doppler ultrasound and digital subtraction angiography (DSA), but Doppler ultrasound is limited by angle dependency and DSA by ionizing radiation. An alternative is the vector flow imaging method based on transverse oscillation (TO), an angle-independent vector velocity technique using ultrasound. In this study, flow complexity and velocity measured with TO were compared with DSA for the assessment of stenosis in the SFA.

Materials and Methods The vector concentration, a measure of flow complexity, and the velocity ratio obtained from the stenosis and a disease-free adjacent vessel segment, were estimated with TO in 11 patients with a total of 16 stenoses of the SFA. TO data were compared with the corresponding stenosis degree percentage obtained with DSA.

Results The correlation between the vector concentration and DSA was very strong $(R=0.93 ; p<0.001 ; 95 \%$ confidence interval $(\mathrm{Cl}): 0.81-0.98)$, while only moderate for velocity ratio and DSA ( $R=0.50 ; \mathrm{p}<0.07 ; 95 \% \mathrm{Cl}: 0.00-0.80)$. The correlation coefficients that were found were significantly different $(\mathrm{p}<0.005)$ without overlapping $\mathrm{Cl}$.

Conclusion The study indicated that flow changes in the SFA induced by stenosis can be quantified with $\mathrm{TO}$, and that stenosis grading may be improved by estimation of flow complexity instead of velocity ratio. TO is a potential diagnostic tool for the assessment of atherosclerosis and peripheral arterial disease.

\section{Introduction}

Peripheral arterial disease (PAD) has an age-adjusted prevalence of 4-15\% and encompasses a wide range of non-coronary arterial pathophysiological processes, which alter the arterial supply to the brain, the visceral organs and the limbs [1,2]. Stenosis of the femoral artery is a disease entity within PAD, and is mainly caused by atherosclerosis [1]. 
Contrast angiography provides detailed information about arterial anatomy and is recommended for the evaluation of patients with stenosis of the femoral artery when revascularization is contemplated. Digital subtraction angiography (DSA) is recommended for contrast angiographic studies, because this technique allows enhanced imaging capabilities compared with conventional unsubtracted contrast angiography and is considered the "gold standard" for defining both normal vascular anatomy and vascular pathology in PAD [1]. However, DSA is invasive, associated with risks of both local and systemic complications, and exposes patients and staff to ionizing radiation [3].

An alternative to DSA is Doppler ultrasound (US) providing peak systolic velocity estimation, velocity ratios within and beyond the stenosis, and evaluation of turbulence $[1,4,5]$. Conventional Doppler US has a major limitation in terms of angle dependency, as only the component of blood velocity directed along the axis of the emitted US beam is measured. Therefore, assumptions of flow direction are necessary for flow quantification, and flow complexity can only be evaluated in terms of flow towards and away from the transducer [6].

The first solutions for angle independent US velocity estimation were proposed several decades ago [7-10]. Later, a promising vector velocity method called transverse oscillation (TO) was proposed by Jensen and Munk [11]. TO provides real-time, angle-independent blood flow estimation, and is currently implemented in commercial scanners as the vector flow imaging (VFI) technique. The TO method has been evaluated for flow estimation of various vessel geometries as reflected in the most recent in-vivo studies [12-16]. However, the TO method has also been investigated for the estimation of cardiac motion $[17,18]$.

Velocity ratios obtained with TO have been used for stenosis assessment in the SFA showing that TO can distinguish between stenoses over and under 50 \% lumen reduction [19]. Another TO-derived parameter for stenosis assessment is vector concentration, a measure of flow complexity, which showed a strong correlation to peak systolic velocities obtained with transesophageal echocardiography (TEE) for flow changes induced by aortic stenosis [20,21].

In this study, patients with stenoses in the SFA were examined with VFI and DSA. The aim of the study was to investigate vector concentration and velocity ratio obtained with TO compared with the stenosis degree percentage obtained with DSA for the assessment of stenoses in the SFA in patients with chronic limb ischemia.

\section{Materials and Methods}

\section{Patients}

The TO and DSA data analyzed in this study are identical to the data used in a previous comparison study of TO and DSA for SFA stenosis, where velocity ratios were calculated from TO vector velocities [19]. Thirty consecutive patients with chronic limb ischemia scheduled for endovascular therapy of the lower extremities were included. Patients were eligible for inclusion if they had one or more previously untreated arteriosclerotic lesions in the SFA. Nineteen patients were excluded due to previous bypass surgery, endovascular surgery, occlusion, no lesions (judged by both US and DSA), or widespread atherosclerotic disease according to The Trans-Atlantic Inter-Society
Consensus Document on Management of Peripheral Arterial Disease [22]. Eleven patients were included with a total of 16 lesions (7 males, 4 females, mean age: 71.6 years, range: $53-84$ years). Written informed consent was obtained from all patients. The local ethics committee waived approval, since US scanning of extremities with PAD is considered a routine procedure (no: $\mathrm{H}-4-2013-001$ ).

\section{Scan setup}

A commercial scanner (UltraView 800, BK Medical, Herlev, Denmark) equipped with a linear transducer using a center frequency of $9 \mathrm{MHz}$ (8670, BK Medical, Herlev, Denmark) was used for the US examinations. All patients were scanned in the angio-suite after 15 min of rest prior to DSA. The US scan included examination of the bifurcation of the common femoral artery down to the level of the SFA, where it enters the adductor canal. Turbulent/disturbed flow was detected in the long-axis view with TO, where vortices and/or sudden aliasing occurred indicating increasing blood flow velocity. The lesion was centered in the scan plane, so flow was estimated within, proximal and distal to the lesion in the same recording ( $\triangleright$ Fig. $\mathbf{1}$ ). A radiodense marker was attached to the skin corresponding to the anatomic location of the TO scan. In the subsequent DSA, the marker pointed directly towards the suspected lesion, ensuring matching ultrasonic and angiographic recordings.

\section{VFI and calculation of vector concentration}

The VFI method estimates both the axial and transverse velocity component using emissions of conventional pulses for Doppler ultrasound. The motion in the axial direction is found as in conventional Doppler ultrasound, while the motion in the transverse direction is found by using a changed apodization in receive beamforming and a special estimator [23]. Previous papers provide detailed explanations of the TO method [11, 23, 24].

The VFI color box was adjusted to cover the vessel with the lesion along with a disease-free adjacent vessel segment included. The adjacent disease-free vessel segment was defined as a segment of the SFA with no narrowing of the lumen and with laminar flow, i. e., without vortices and/or sudden aliasing. The pulse repetition frequency (PRF) was adjusted for each scan ensuring optimal filling of the vessel in both the stenosed and the adjacent disease-free vessel segment, even if aliasing in systole occurred. Wall filter and color gain were likewise adjusted to the level providing optimal filling of the vessel without flow artifacts outside the vessel. All other settings remained in default mode. The angle of insonation was 70-90 degrees in all cases. The temporal resolution of the TO estimation was 16 frames/s, and the maximum scan depth was approximately $5 \mathrm{~cm}$ due to the available transducer setup. The recorded TO cine loop of $14 \mathrm{~s}$ corresponded to 225 frames. An overview of the applied scan settings along with TO acquisition parameters is given in > Table 1. On the US scanner, vector velocity estimates were displayed in real-time, but without any quantification of velocities available. Afterwards, the cine loops were analyzed off-line using MATLAB (MathWorks, Natick, MA, USA) as previously described [25].

The averaged vector concentration $r$ of the blood flow in the SFA during 5 consecutive systoles from the beginning of the recorded cine loop was calculated for each examined vessel segment. The region of interest (ROI) used for vector concentration estimation 

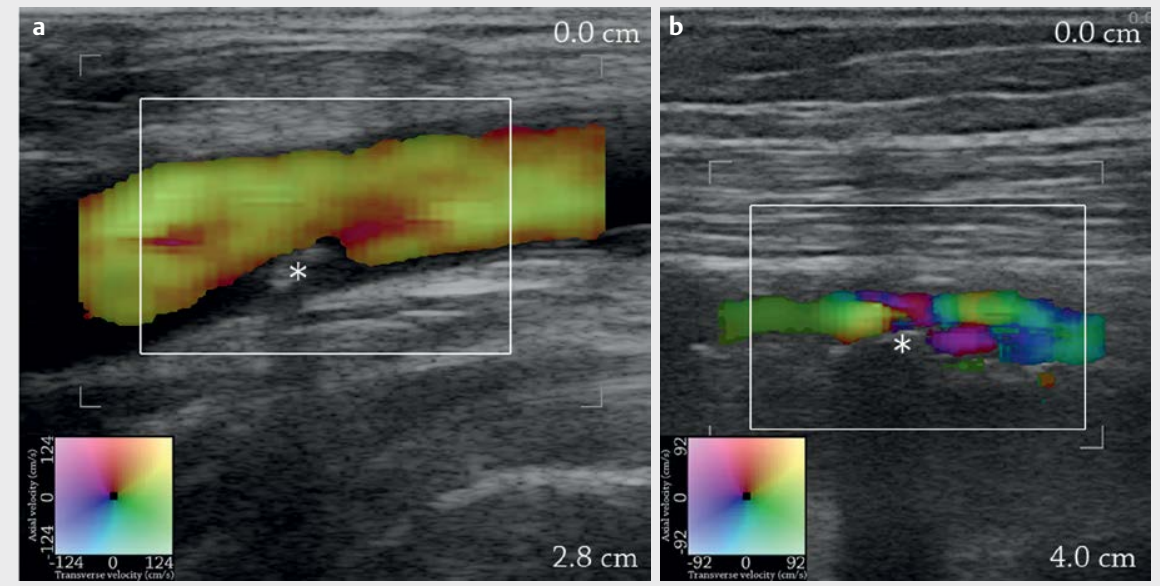

-Fig. 1 Vector velocity images of 2 patients with stenosis of the SFA are shown in $\mathbf{a}$ and $\mathbf{b}$, where A corresponds to lesion no. 3 and B to lesion no. 1 ( $\vee$ Table 1). Both frames are taken from systole. The lesions are marked with an asterisk, and in each frame, the ROI for calculation of the vector concentration is illustrated with a white box. Direction and velocity of the blood flow estimated with TO are shown by the color map.

- Table 1 TO acquisition setup and scan settings with standard deviation (SD) in parentheses.

\begin{tabular}{|l|l|}
\hline Number of elements & $\mathbf{1 2 8}$ \\
\hline Bandwidth & $70 \%$ \\
\hline Pitch & $0.3 \mathrm{~mm}$ \\
\hline Kerf & $0.035 \mathrm{~mm}$ \\
\hline Height & $4 \mathrm{~mm}$ \\
\hline Elevation focus & $20 \mathrm{~mm}$ \\
\hline Pulse length & 6 cycles sinusoidal \\
\hline Lateral wavelength & $4^{*}$ pitch \\
\hline Average PRF & $3.3 \mathrm{kHz}(1.6 \mathrm{kHz})$ \\
\hline Average wall filter cutoff frequency & $134.8 \mathrm{~Hz}(73.1 \mathrm{~Hz})$ \\
\hline Average gain & $52.3 \%(3.6 \%)$ \\
\hline
\end{tabular}

included the lesion along with the disturbed flow in the periphery of the lesion ( $>$ Fig. 1). The vector concentration is a calculation of the vector angle spread within the ROI, and a measure of flow complexity [26]. In brief, the vector concentration is calculated as follows. For each position $\mathrm{i}$ in the vector map, where the axial velocity component $v_{x}$ and the transverse velocity component $v_{z}$ are estimated, the flow angle $\theta_{i}$ of the vector is:

$\theta_{i}=\arctan \left(v_{x, i}, v_{z, i}\right)$

Each flow angle $\theta_{i}$ is represented on the unit circle as $\mathrm{P}_{i}=\left(\mathrm{x}_{i}, \mathrm{y}_{i}\right)$, where $x_{i}=\cos \left(\theta_{i}\right)$ and $y_{i}=\sin \left(\theta_{i}\right)$. For each ROI encompassing the entire vessel, the mean value for $x_{i}$ and $y_{i}$ is found as,

$\bar{x}=\frac{1}{n} \sum_{i=1}^{n} \cos \left(\theta_{i}\right)$ $\bar{y}=\frac{1}{n} \sum_{i=1}^{n} \sin \left(\theta_{i}\right)$

To quantify the flow complexity, i. e., the vector angle spread, the vector concentration $r$ is found using Pythagoras' theorem:

$r=\sqrt{\bar{x}^{2}+\bar{y}^{2}}$

where $r$ is one for perfect laminar flow, and decreases towards zero with increased complex flow. Thus, vector concentration can be regarded as an in-vivo measure of flow complexity comparable to the Reynolds number, which is used to predict flow patterns in fluid mechanics [27].

Furthermore, from each TO recording, 3 frames illustrating flow with the best possible filling of the vessel in both the lesioned and the healthy part of the SFA were selected. The velocity ratio calculated from TO estimates of each stenosis was found as the maximum velocity detected centrally in the lesioned segment divided by the maximum velocity detected centrally in the adjacent disease-free segment. The frames were obtained from the cardiac cycle, where flow in both the stenosed and adjacent disease-free vessel segment was antegrade and without aliasing. The velocity ratios were not calculated at identical time points in the cardiac cycle. However, a constant velocity ratio for each stenosis was assumed, when the velocities used for the calculation of the velocity ratio were acquired from the same frame, i.e., at the same time point [19]. The velocity ratios reported in this paper are identical to the velocity ratios found in a previous paper, where a more thorough explanation of the velocity ratio estimation is given [19].

The calculations of vector concentration and velocity ratio were performed separately and by 2 different experienced radiologists (KLH and $\mathrm{PMH}$ ) blinded to the corresponding results of the DSA.

\section{Angiography}

An Infinix-i system (model INFX-8000V, Toshiba Medical Systems Corporation, Tochigi-ken, Japan) was used for DSA. An 11-cm 5-Fr 
sheath was placed in the artery. A 4- or 5-Fr catheter was used when needed for contrast injections. DSA of the femoral artery was performed using 2 frames $/ \mathrm{s}$ and a $6-10 \mathrm{ml}$ iodine contrast injection (Visipaque $270 \mathrm{mgl} / \mathrm{ml}$, GE Healthcare). Routine anteroposterior images in one plane were recorded and occasionally supplemented by oblique projections. Subsequent measurements were performed on a standard workstation. The DSA image yielding the most severe diameter reduction was used for calculation of the stenosis degree percentage, i. e., the smallest diameter in the stenosis vs. the diameter in an adjacent normal arterial segment. An experienced radiologist not otherwise involved in this study and blinded to the result of the corresponding US scan, calculated the stenosis degree percentage for each patient.

\section{Statistical analysis}

Measurements obtained with TO were initially analyzed with descriptive statistics, i. e., mean and standard deviation (SD). The TO measurements, i. e., vector concentration and velocity ratio, were then compared with the stenosis degree percentage obtained with DSA using linear regression analyses with a 2-tailed significance value and $\mathrm{p}<0.05$ considered significant. The correlation coefficient, regression equation, and confidence interval $(\mathrm{Cl})$ using Fisher's r-to-z-transformation were calculated. Statistical analyses were performed with IBM SPSS Statistics v. 19 (SPSS Inc., Chicago, IL, USA).

\section{Results}

The descriptive statistics on vector concentration and velocity ratio with the corresponding stenosis degree percentage are given in - Table 2. The mean vector concentration and the mean velocity ratio obtained with TO were 0.67 (SD: 0.21 ) and 1.25 (SD: 0.35), re- spectively, and the mean stenosis degree percentage obtained with DSA was 33.6\% (SD: $27.8 \%$ ). The association between vector concentration and stenosis degree percentage, and between velocity ratio and stenosis degree percentage are illustrated with scatterplots in $\mathbf{F i g . ~ 2 . ~ F o r ~ t h e ~ c o m p a r i s o n ~ b e t w e e n ~ v e c t o r ~ c o n c e n t r a t i o n ~ a n d ~}$ DSA, the correlation coefficient $R$ was 0.93 ( $p<0.001 ; 95 \% \mathrm{Cl}: 0.81$ to 0.98 ), and the regression equation was $y=-0.007 x+0.917$, where $x$ corresponded to the stenosis degree percentage and $y$ to the vector concentration. For the comparison between velocity ratio and DSA, the correlation coefficient R was 0.50 ( $p<0.07 ; 95 \% \mathrm{Cl}: 0.00$ to 0.80 ), and the regression equation was $y=-0.012 x+1.210$, where $x$ corresponded to the stenosis degree percentage and $y$ to the velocity ratio. The correlation coefficients for the 2 comparison analyses were significantly different $(\mathrm{p}<0.005)$ without overlapping $\mathrm{Cl}$.

\section{Discussion}

This study of stenosis assessment in the SFA indicated that vector concentration was more strongly associated than velocity ratio to stenosis degree percentage as the R-value was higher without overlapping $\mathrm{Cl}$ for corresponding correlation analyses. The presented results support previous studies of vector concentration showing excellent performance in the evaluation of aortic valve stenosis with intraoperative $\mathrm{VFI}$ of blood flow in the ascending aorta $[20,21,28]$. The 2 most recent studies showed that the flow complexity quantified with vector concentration was different among patients with a normal, stenosed, and replaced aortic valve $(p<0.0001)$, and with a strong association to peak systolic velocity $(p<0.0001, R=0.87$ and 0.88$)[20,21]$.

Flow complexity can be assessed in conventional US using estimation of spectral broadening in spectral Doppler, power intensity

- Table 2 Averaged vector concentration and velocity ratio with standard deviation (SD) in parentheses along with the corresponding DSA-derived stenosis degree percentage for each stenosis examined.

\begin{tabular}{|c|c|c|c|c|c|}
\hline Patient no. & Lesion no. & $\begin{array}{l}\text { Shadowing calcifications } \\
\text { (yes/no) }\end{array}$ & $\begin{array}{l}\text { Velocity ratio } \\
\text { (SD) }\end{array}$ & $\begin{array}{l}\text { Vector concentration } \\
\text { (SD) }\end{array}$ & $\begin{array}{l}\text { Stenosis degree } \\
\text { percentage [\%] }\end{array}$ \\
\hline 1 & 1 & $N$ & $2.2(0.41)$ & $0.35(0.04)$ & 78 \\
\hline 1 & 2 & $Y$ & $1.1(0.15)$ & $0.94(0.03)$ & 0 \\
\hline 2 & 3 & $\mathrm{~N}$ & $1.0(0.06)$ & $0.96(0.03)$ & 0 \\
\hline 2 & 4 & $\mathrm{Y}$ & $1.2(0.06)$ & $0.73(0.06)$ & 19 \\
\hline 3 & 5 & $N$ & $2.9(0.58)$ & $0.41(0.06)$ & 68 \\
\hline 4 & 6 & $N$ & $2.6(1.59)$ & $0.57(0.04)$ & 65 \\
\hline 5 & 7 & $\mathrm{Y}$ & $1.2(0.15)$ & $0.79(0.03)$ & 37 \\
\hline 5 & 8 & Y & $0.9(0.32)$ & $0.81(0.05)$ & 31 \\
\hline 6 & 9 & Y & $2.1(0.30)$ & $0.66(0.01)$ & 33 \\
\hline 6 & 10 & Y & $1.5(0.15)$ & $0.82(0.09)$ & 15 \\
\hline 6 & 11 & Y & $1.2(0.15)$ & $0.75(0.13)$ & 15 \\
\hline 7 & 12 & $N$ & $2.2(0.17)$ & $0.40(0.07)$ & 62 \\
\hline 8 & 13 & $\mathrm{Y}$ & $2.5(0.55)$ & $0.71(0.08)$ & 11 \\
\hline 9 & 14 & $N$ & $1.2(0.10)$ & $0.48(0.17)$ & 47 \\
\hline 10 & 15 & $N$ & $1.3(0.06)$ & $0.95(0.02)$ & 0 \\
\hline 11 & 16 & $\mathrm{~N}$ & $1.0(0.06)$ & $0.38(0.09)$ & 67 \\
\hline
\end{tabular}



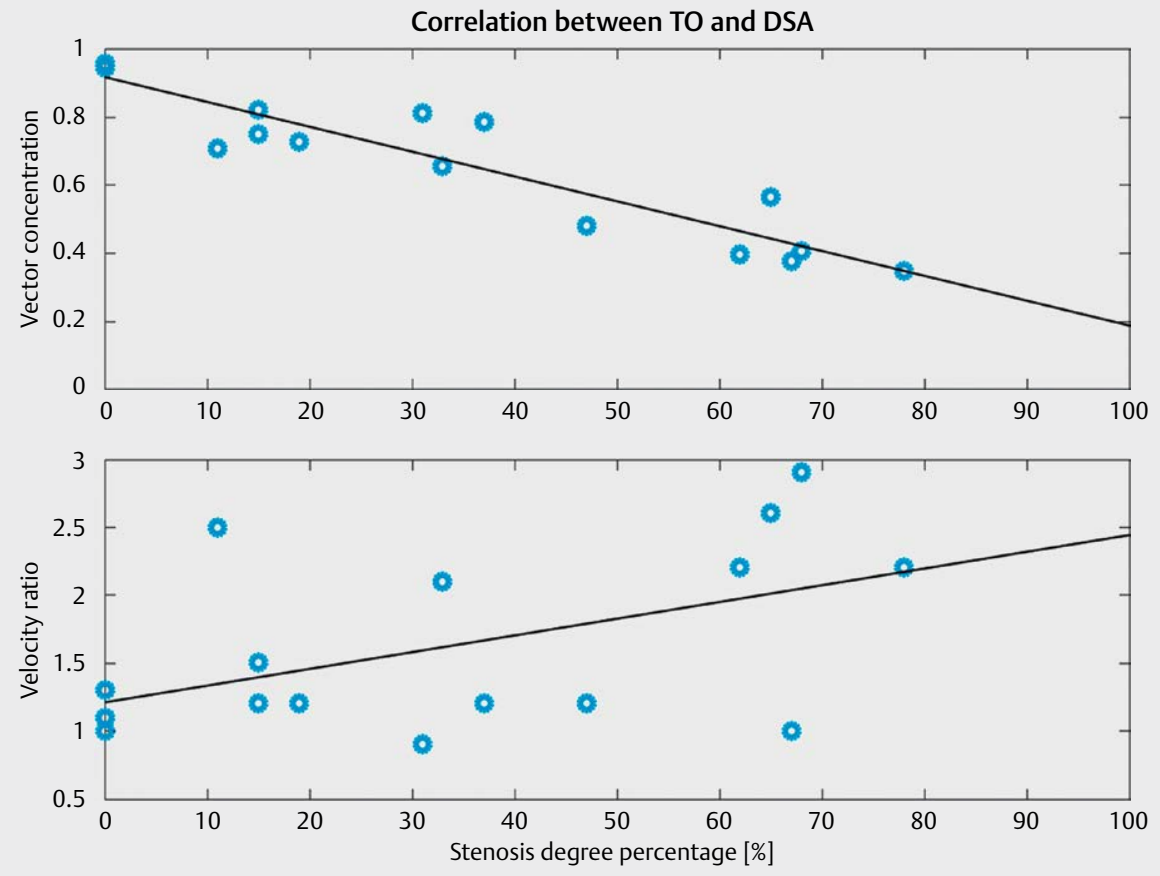

- Fig. 2 Scatterplots of TO-derived vector concentration and velocity ratio compared with DSA-derived stenosis degree percentage. Line of best fit is illustrated with a black solid line for each subplot.

in power Doppler, or by evaluation of mosaic patterns using color Doppler [29-31]. However, stenosis grading in conventional US is normally done by velocity estimation, such as measurements of peak velocities and mean gradients in aortic valve stenosis, and velocity ratios in stenosis of the SFA [5, 32].

The TO data in this paper have previously been used for velocity ratio estimation showing that TO was able to separate stenosis over and under $50 \%$ lumen reduction $(p<0.01)$. Two patients were considered outliers (patient 8 and 11) [19]. To test performance, linear correlation analysis was done for both velocity ratio and vector concentration compared with DSA in this study. So, applying the same TO data for evaluation of vector concentration instead of velocity ratio gave an improved association to the same data set of DSA-derived stenosis degree percentage, and without any outliers. Therefore, vector concentration may be a better parameter for the evaluation of stenosis and the resultant flow changes in the SFA than velocity ratio estimation when using vector velocity data.

There are several reasons for this improvement. TO is based on conventional pulsed US emission, and therefore is limited by aliasing like conventional Doppler US. However, vector concentration estimation is less PRF-sensitive than velocity estimation. While aliased laminar blood flow will result in velocity estimates with large errors, the flow direction will be reversed but uniform, and therefore, have a less affected vector concentration [21, 28]. As previously stated, some reduction in the vector concentration estimate has to be expected when aliasing occurs even for laminar flow, as the central part of the flow with the highest and aliased velocities will appear retrograde, whereas the peripheral flow will remain antegrade [21]. Thus, at least 2 opposing flow angles within the ROI will be present, which will increase the vector angle spread of the $\mathrm{ROI}$, and in the case with an equal amount of scatter moving in 2 opposite directions, the vector concentration will approach zero. Nevertheless, this effect is less prominent than the effect of the actual increase in flow complexity for increasing vessel stenosis. This has been indicated in previous papers, where TO-derived vector concentration estimates compared with peak systolic velocities obtained with continuous wave US using TEE had a strong linear relationship, even when including vector concentration estimates obtained above the Nyquist limit [20,21]. In the previous papers evaluating vector concentration for aortic valve stenosis, the PRF was adjusted to the peak systolic velocity to reduce aliasing, while the PRF in this study was adjusted to optimal filling of the lesioned and adjacent disease-free vessel segment even if aliasing occurred. This underlines the need for a more thorough evaluation of vector concentration in relation to PRF settings.

Another advantage of vector concentration estimation is less dependency on peak flow alignment. The blood flow in-vivo is often non-parabolic, and therefore, the highest velocities are not always found in the center of the lumen but along the vessel wall as shown in a previous study with VFI and MRI of flow in the carotid artery [33]. Also, a VFI study of blood flow in the ascending aorta showed only a moderate association with TEE for peak systolic velocity estimation, and a weak association with pulmonary artery catheter thermodilution for cardiac output estimation, mainly due to asymmetry of the aortic flow [34]. In vector concentration estimation, only the direction of flow is used. If the blood flow is laminar, it will be uniform even for off-center evaluation, and likewise, if the blood flow is complex, it will behave complex for off-center evaluation. 
Thus, it is not crucial to capture the jet of the flow, as all blood flow layers are representative of the flow complexity reflecting the degree of stenosis.

The angle-independent velocity estimation in TO is an obvious improvement compared with blood flow estimation in conventional US systems, as flow direction and velocity to every pixel within an ROI are given. In vector concentration estimation, all flow data within the ROI are used providing much more data for the evaluation when compared with the velocity estimation, whether obtained with conventional Doppler US or VFI.

The reduced dependency of flow alignment and the use of more flow data are an advantage when stenoses are calcified creating acoustic shadowing. A previous study of carotid artery stenosis showed that in the presence of calcifications, spectral Doppler US is inadequate to accurately determine the degree of stenosis when compared with computed tomographic angiography [35]. According to $>$ Table $2,50 \%$ of the included patients in this study had shadowing calcifications in the SFA stenosis, and vector concentration still correlated well with the corresponding stenosis degree percentage. Moreover, the 2 patients ( 8 and 11) acting as outliers for velocity ratio estimation both had calcifications ( Table 2 ).

VFI estimation is probably less user-dependent and more robust than default velocity estimation as angle correction and range gate positioning are avoided. Two recent papers indicated that VFI is more precise and accurate and less angle- and operator-dependent than conventional spectral Doppler US for in-vivo velocity estimation $[12,13]$. With the introduction of vector concentration, VFI may also prove to be less PRF-dependent as indicated by this and previous papers $[20,21,28]$.

This study had several limitations. First of all, the study population was small. Also, vector concentration was not calculated directly on the scanner but processed off-line. However, the calculations were not computationally demanding, and should, therefore, be easy to implement on the commercial platform for real-time estimation. The vector concentration estimation could be further improved with a more automated and less user-dependent interface, where predefined scan and ROI settings guide the user. Only one operator performed the examinations in this study, hence, no interobserver variability was assessed. Inter- and intraobserver variability of velocity estimation has been investigated for VFI and revealed superior performance compared with spectral Doppler US [13]. DSA is a 2D method, and underestimation of stenoses can therefore occur if the smallest diameter of the vessel is not shown in the angiographic projection. DSA is occasionally supplemented by oblique projections if any doubt about a stenosis is raised, but that is no guarantee for a projection illustrating the most severe stenosis degree. In > Fig. 1a, a stenosis shown with US corresponded to a stenosis degree percentage of $0 \%$ according to DSA ( $\triangleright$ Table 2 ) illustrating this limitation.

In this study, spectral Doppler was not used as a reference, as DSA is considered the gold standard for diagnosing and grading PAD. However, in future studies of vector concentration obtained with VFI, spectral Doppler will be employed as a reference method for a more complete validation. The optimal parameter settings for vector concentration estimation, e. g., PRF, gain and wall filter, should be investigated in a controlled setup, and flow disturbances in other vessel geometries, e. g., stenosis of the carotid artery, in arteriovenous fistula, and of the valves of the heart using vector concentration should also be explored. The studies should include larger patient populations and more US operators. The TO-derived vector concentration could be a potential powerful clinical tool for a reliable, easy-to-use, fast, and non-ionizing method of stenosis assessment in patients suffering cardiovascular diseases such as PAD.

\section{Conclusion}

Vector concentration is a new parameter for blood flow evaluation obtained with angle-independent vector velocity estimation. In this study of PAD in the SFA, vector concentration was superior to velocity ratio for stenosis assessment when compared with stenosis degree percentage obtained with DSA. The study indicates that vector concentration could be a better parameter for stenosis evaluation than evaluation of blood flow velocities when using VFI.

\section{Conflict of Interest}

J.A.J. developed and patented the Transverse Oscillation technique and earns royalties from BK Ultra- sound for the sale of scanners with the Transverse Oscillation Vector Flow Imaging technique implemented.

\section{References}

[1] Hirsch AT, Haskal Z], Hertzer NR. ACC/AHA 2005 Practice guidelines for the management of patients with peripheral arterial disease (lower extremity, renal, mesenteric, and abdominal aortic). Circulation 2006; 113: e463-e654

[2] Selvin E, Erlinger TP. Prevalence of and risk factors for peripheral arterial disease in the United States: Results from the National Health and Nutrition Examination Survey, 1999-2000. Circulation 2004; 110: 738-743

[3] Egglin TK, O'Moore PV, Feinstein AR et al. Complications of peripheral arteriography: A new system to identify patients at increased risk. J Vasc Surg 1995; 22: 787-794

[4] Flanigan DP, Ballard JL, Robinson D et al. Duplex ultrasound of the superficial femoral artery is a better screening tool than ankle-brachial index to identify at risk patients with lower extremity atherosclerosis. J Vasc Surg 2008; 47: 789-792; discussion 92-93

[5] Khan SZ, Khan MA, Bradley B et al. Utility of duplex ultrasound in detecting and grading de novo femoropopliteal lesions. J Vasc Surg 2011; 54: 1067-1073

[6] Hoskins PR, Kenwright DA. Recent developments in vascular ultrasound technology. Ultrasound 2015; 23: 158-165

[7] Bonnefous O. Measurement of the complete (3D) velocity vector of blood flows. Proc IEEE Ultrason Symp 1988; 795-799

[8] Fox MD. Multiple crossed-beam ultrasound doppler velocimetry. IEEE Trans Son Ultrason 1978; 25: 281-286

[9] Newhouse VL, Censor D, Vontz T et al. Ultrasound doppler probing of flows transverse with respect to beam axis. IEEE Trans Biomed Eng 1987; 34: 779-788

[10] Trahey GE, Allison JW, Ramm OT. Angle independent ultrasonic detection of blood flow. IEEE Trans Biomed Eng 1987; 34: 965-967

[11] Jensen JA, Munk P. A new method for estimation of velocity vectors. IEEE Trans Ultrason Ferroelect Freq Control 1998; 45: 837-851 
[12] Brandt AH, Hansen KL, Ewertsen C et al. A comparison study of vector velocity, spectral doppler and magnetic resonance of blood flow in the common carotid artery. Ultrasound Med Biol 2018; 44: 1751-1761

[13] Brandt AH, Moshavegh R, Hansen KL et al. Vector flow imaging compared with pulse wave doppler for estimation of peak velocity in the portal vein. Ultrasound Med Biol 2018; 44: 593-601

[14] Hansen KL, Juul K, Moller-Sorensen H et al. Pediatric transthoracic cardiac vector flow imaging - A preliminary pictorial study. Ultrasound International Open 2018; 5: E20-E26

[15] Bechsgaard T, Hansen KL, Brandt AH et al. Evaluation of peak reflux velocities with vector flow imaging and doppler ultrasound in varicose veins. Ultrasound International Open 2018; 4: E91-E98

[16] Lenge M, Ramalli A, Tortoli P et al. Plane-wave transverse oscillation for high-frame-rate 2-D vector flow imaging. IEEE Trans Ultrason Ferroelectr Freq Control 2015; 62: 2126-2137

[17] Alessandrini M, Basarab A, Boussel L et al. A new technique for the estimation of cardiac motion in echocardiography based on transverse oscillations: A preliminary evaluation in silico and a feasibility demonstration in vivo. IEEE Trans Med Imaging 2014; 33: 1148-1162

[18] Varray F, Liebgott H. Multi-resolution transverse oscillation in ultrasound imaging for motion estimation. IEEE Trans Ultrason Ferroelectr Freq Control 2013; 60: 1333-1342

[19] Hansen PM, Hansen KL, Pedersen MM et al. Arteriosclerotic Lesions in the Superficial Femoral Artery (SFA) characterized with velocity ratios using vector velocity ultrasound. Ultrasound International Open 2018; 4: E79-E84

[20] Hansen KL, Moller-Sorensen H, Kjaergaard J et al. Intra-operative vector flow imaging using ultrasound of the ascending aorta among 40 patients with normal, stenotic and replaced aortic valves. Ultrasound Med Biol 2016; 42: 2414-2422

[21] Hansen KL, Moller-Sorensen H, Kjaergaard J et al. Aortic valve stenosis increases helical flow and flow complexity: A study of intra-operative cardiac vector flow imaging. Ultrasound Med Biol 2017; 43: 16071617

[22] Norgren L, Hiatt WR, Dormandy JA et al. Inter-Society Consensus for the Management of Peripheral Arterial Disease (TASC II). J Vasc Surg 2007; 45: Suppl S S5-S67
[23] Jensen JA. A new estimator for vector velocity estimation. IEEE Trans Ultrason Ferroelec Freq Contr 2001; 48: 886-894

[24] Udesen J, Jensen JA. Investigation of transverse oscillation method. IEEE Trans Ultrason Ferroelec Freq Contr 2006; 53: 959-971

[25] Pedersen MM, Pihl MJ, Haugaard P et al. Comparison of real-time in vivo spectral and vector velocity estimation. Ultrasound Med Biol 2012; 38: 145-151

[26] Pedersen MM, Pihl M], Haugaard P et al. Novel flow quantification of the carotid bulb and the common carotid artery with vector flow ultrasound. Ultrasound Med Biol 2014; 40: 2700-2706

[27] Reynolds O. An experimental investigation of the circumstances which determine whether the motion of water shall be direct or sinuous and of the law of resistance in parallel channels. Philos Trans R Soc Lond 1883; 174: 935-987

[28] Hansen KL, Moller-Sorensen H, Pedersen MM et al. First report on intraoperative vector flow imaging of the heart among patients with healthy and diseased aortic valves. Ultrasonics 2015; 56: 243-250

[29] Hutchison SJ, Rosin BL, Curry S et al. Transesophageal echocardiographic assessment of lesions of the right ventricular outflow tract and pulmonic valve. Echocardiography 1996; 13: 21-34

[30] Cloutier G, Allard L, Durand LG. Changes in ultrasonic doppler backscattered power downstream of concentric and eccentric stenoses under pulsatile flow. Ultrasound Med Biol 1995; 21: 59-70

[31] Stringer DA, O'Halpin D, Daneman A et al. Duplex doppler sonography for renal artery stenosis in the post-transplant pediatric patient. Pediatr Radiol 1989; 19: 187-192

[32] Manning W]. Asymptomatic aortic stenosis in the elderly: A clinical review. JAMA 2013; 310: 1490-1497

[33] Hansen KL, Udesen J, Thomsen C et al. In vivo validation of a blood vector velocity estimator with MR angiography. IEEE Trans Ultrason Ferroelect Freq Control 2009; 56: 91-100

[34] Hansen KL, Moller-Sorensen H, Kjaergaard J et al. Vector flow imaging compared with conventional doppler ultrasound and thermodilution for estimation of blood flow in the ascending aorta. Ultrason Imaging 2015; 1-16

[35] Mohebali ], Patel VI, Romero JM et al. Acoustic shadowing impairs accurate characterization of stenosis in carotid ultrasound examinations. J Vasc Surg 2015; 62: 1236-1244 